\title{
Avaliação dos aspectos físicos e parâmetros de normalidade da radiografia de tórax por estudantes de medicina em faculdade de Teresina-PI
}

\author{
Evaluation of physical aspects and standards of normality of thorax radiography by medical \\ students in faculty of Teresina-PI \\ Evaluación de aspectos físicos y estándares de normalidad de la radiografía de tórax por \\ estudiantes de médico en facultad de Teresina-PI
}

Recebido: 08/12/2021 | Revisado: 12/12/2021 | Aceito: 14/12/2021 | Publicado: 22/12/2021

Yago Reis de Deus Barros
ORCID: https://orcid.org/0000-0003-1178-208X
Centro Universitário Uninovafapi, Brasil
E-mail: yagoreis2009@hotmail.com
Francisco Frazão Mendes Junior
ORCID: https://orcid.org/0000-0002-7613-5409
Centro Universitário Uninovafapi, Brasil
E-mail: frazaojunior.fj@gmail.com
Williams Cardec da Silva
ORCID: https://orcid.org/0000-0002-7167-4876
Centro Universitário Uninovafapi, Brasil
E-mail: willcardec@ @otmail.com

\begin{abstract}
Resumo
O raio x de tórax é um dos exames de imagem mais solicitados na prática médica, é a partir dele que é possível identificar as principais estruturas torácicas. Entretanto, além de possuir uma leitura difícil, a interpretação deste depende do operador do exame, da técnica utilizada e do conhecimento do avaliador. A pesquisa que consiste em um estudo transversal, descritivo e de abordagem quantitativa, foi realizada através de um formulário de 9 perguntas elaborado pelos próprios autores e respondido por acadêmicos do $9^{\circ}$ ao $12^{\circ}$ período de medicina da instituição UNINOVAFAPI, em Teresina-PI, no ano de 2021, e tem como objetivo central avaliar o conhecimento dos estudantes de medicina sobre os parâmetros técnicos e de normalidade desse exame de imagem. Os resultados mostraram uma média aritmética de 6 pontos, que correspondia a $66,7 \%$ do questionário. Constatou-se que $71,33 \%$ dos estudantes atingiram uma nota igual ou superior à média do exame, portanto, conclui-se que a pesquisa encontrou um resultado satisfatório. No presente estudo, os resultados apresentam índices relevantes na avaliação dos parâmetros de normalidade da radiografia de tórax por estudantes de medicina. Evidencia-se que o ensino apresenta uma grande variabilidade entre os programas de treinamento em residência médica e faculdade de medicina.
\end{abstract}

Palavras-chave: Radiografia torácica; Estudantes de medicina.

\begin{abstract}
The chest $\mathrm{x}$-ray is one of the most requested imaging exams in medical practice, it is from this that it is possible to identify the main thoracic structures. However, in addition to being difficult to read, its interpretation depends on the exam operator, the technique used and the evaluator's knowledge. The research, which consists of a cross-sectional, descriptive study with a quantitative approach, was carried out using a 9-question form prepared by the authors themselves and answered by academics from the 9th to the 12th period of medicine at the UNINOVAFAPI institution, in Teresina-PI, in the year of 2021, and its main objective is to assess the knowledge of medical students about the technical parameters and normality of this imaging exam. The results showed an arithmetic mean of 6 points, which corresponded to $66.7 \%$ of the questionnaire. It was found that $71.33 \%$ of students achieved a grade equal to or higher than the average of the exam, therefore, it is concluded that the research found a satisfactory result. In the present study, the results show relevant indices in the assessment of normality parameters of chest X-rays by medical students. It is evident that teaching presents great variability between training programs in residency and medical school.
\end{abstract}

Keywords: Radiography, thoracic; Students, medical. 


\begin{abstract}
Resumen
La radiografía de tórax es uno de los exámenes de imagen más solicitados en la práctica médica, es a partir de ella que es posible identificar las principales estructuras torácicas. Sin embargo, además de ser difícil de leer, su interpretación depende del operador del examen, la técnica utilizada y los conocimientos del evaluador. La investigación, que consiste en un estudio descriptivo transversal con enfoque cuantitativo, se realizó mediante un formulario de 9 preguntas elaborado por los propios autores y respondido por académicos del $9^{\circ}$ al $12^{\circ}$ período de medicina de la institución UNINOVAFAPI. en Teresina-PI, en el año 2021, y su principal objetivo es evaluar el conocimiento de los estudiantes de medicina sobre los parámetros técnicos y la normalidad de este examen de imagen. Los resultados arrojaron una media aritmética de 6 puntos, lo que correspondió al 66,7\% del cuestionario. Se encontró que el $71.33 \%$ de los estudiantes logró una calificación igual o superior al promedio del examen, por lo que se concluye que la investigación encontró un resultado satisfactorio. En el presente estudio, los resultados muestran índices relevantes en la evaluación de los parámetros de normalidad de la radiografía de tórax por estudiantes de medicina. Es evidente que la docencia presenta una gran variabilidad entre los programas de formación en residencia y la facultad de medicina.
\end{abstract}

Palabras clave: Radiografía torácica; Estudiantes de medicina.

\title{
1. Introdução
}

A radiografia torácica é um dos exames de imagem mais solicitados na prática médica. O estudo radiológico do tórax possibilita ao profissional bem capacitado a avaliação, por meio do contraste natural, de estruturas como a trama pulmonar, pulmões, pleuras, coração, estruturas da via aérea superior, parede torácica, abdome superior, esôfago e, assim, o diagnóstico, monitoração e acompanhamento de inúmeras patologias (Chen et al., 2012).

Contudo, o exame apresenta uma limitação no que tange à representação fidedigna dos componentes do tórax, principalmente, por representar uma imagem bidimensional de estruturas tridimensionais, o que interfere tanto na qualidade quanto especificidade do exame. Logo, há uma correlação entre a qualidade da técnica utilizada, que é operador dependente e, do conhecimento técnico e clínico de quem interpreta a radiografia torácica (Wada et al., 2019b).

Assim, o estudo da radiografia é essencial para o discernimento da qualidade e validade da imagem que está sendo avaliada clinicamente. Exigindo, portanto, conhecimento acerca dos parâmetros técnicos como inspiração correta, penetração do RX (raio x) e alinhamento, além da rotina semiológica realizada de forma sistemática para que haja interpretação mais assertiva possível do exame (Wada et al., 2019b).

Dessa forma, tendo em vista a relevância do conhecimento sobre radiografia de tórax em diversos âmbitos da prática médica, entende-se que é fundamental para a formação dos estudantes o estudo radiológico no período da graduação. Por conseguinte, o objeto do nosso estudo é avaliação dos parâmetros de normalidade da radiografia de tórax por estudantes de medicina.

O interesse pelo estudo do tema se deu pela importância que a radiografia de tórax tem na rotina médica, tanto por ser um dos exames de imagem mais realizados no mundo quanto pela relevância clínica que ele possui. Ainda assim, possui alta margem de erro, pois mesmo sendo um exame simples, requer um treinamento específico do olhar clínico, tornando um exame difícil de ser estudado (Cerri et al., 2017).

Nesse sentido, é evidente que a formação que contempla de forma efetiva o estudo e treinamento radiológico refletirá em profissionais mais capacitados para diagnosticar, tratar e dar seguimento a diversas patologias através da interpretação da radiografia torácica associada à clínica do paciente. Dessa forma, é importante que haja uma melhor capacitação dos estudantes durante a graduação, visando o reconhecimento dos padrões de normalidades para que haja maior precisão diante de possíveis alterações radiológicas encontradas durante o exercício da profissão (Geel et al., 2019).

Portanto, o estudo tem por finalidade avaliar o conhecimento individual desses estudantes, além da possibilidade de alertar e despertar interesse acerca do tema. Outrossim, o trabalho final será entregue à coordenação do curso de medicina do Uninovafapi, o qual poderá contribuir com a maior ênfase desse assunto na grande curricular da faculdade e, consequentemente, na formação de profissionais mais qualificados para o exercício da boa prática médica. Finalmente, o objetivo da pesquisa é 
avaliar o conhecimento dos estudantes de medicina sobre os parâmetros técnicos e de normalidade da radiografia de tórax, bem como causas que dificultem esta identificação.

\section{Referencial Teórico}

\subsection{Introdução a Radiologia}

Em 1895, o físico de origem alemã, Wilhelm Conrad Röntgen, descobriu uma radiação que atravessava objetos opacos, não era visível e gerava uma impressão em placas de fotografia da época. Portanto as chamou de "raios X", uma vez que esta era diferente de outras radiações já conhecidas em seu período (Salvador et al., 2018).

Essa descoberta, gerou impacto em diversas áreas. Uma das principais foi no contexto médico, uma vez que inúmeros avanços foram alcançados, como por exemplo os exames de imagens que são realizados no dia de hoje (Salvador et al., 2018).

$\mathrm{O}$ raio $\mathrm{x}$ consiste em uma radiação criada em um tubo a vácuo, em que são colocados 2 eletrodos, 1 cátodo, que corresponde ao polo positivo e 1 ânodo, que corresponde ao polo negativo. Uma corrente elétrica é fornecida ao cátodo e faz com que elétrons se desloquem do cátodo em direção ao ânodo. (Massoni et al., 2017).

Por fim, uma vez que os elétrons se choquem com o ânodo, é produzido uma energia, conhecida como raio X, e buscando documentar a captura dessa radiação, foram utilizadas placas fotográficas da época (Salvador et al., 2018).

Com base nisso, o pioneiro na identificação destes raios, decidiu avaliar a penetração dos mesmos, começando com objetos opacos e evoluindo até a ideia de utilizar a mão de sua própria esposa. Neste último caso, foi observado que a radiação era mais absorvida pelos ossos, contrastando com os outros tecidos da mão (Salvador et al., 2018).

As imagens de raio $\mathrm{X}$ são formadas pela radiação que passa pelas estruturas do corpo e chegam até um filme fotossensível. Ao ser atingido, ocorrem reações, produzindo colorações preta, diferentes tonalidades de cinza e branca. Tais nuances de cor deve-se as diferentes capacidades dos tecidos do corpo de absorver radiação, quanto mais absorção houver, mais clara será pigmentação (Marchiori \& Santos, 2015).

Entretanto, essa energia gerada pela radiografia, tem efeitos nocivos a saúde humana uma vez que ela possui a capacidade de ionizar células do corpo, podendo até gerar estruturas cancerígenas a depender da quantidade de radiação, quantidade de vezes aplicada e duração (Nascimento, 2018).

Com isso, várias modificações foram realizadas nas máquinas de radiografia. Alterações estas, que possibilitaram um menor tempo na realização do exame, a partir do controle de intensidade dos raios, diminuindo o tempo de exposição e consequentemente possíveis problemas relacionados. Outras alterações também tem sua importância, tais como a redução da área radiografada, dosímetros para maior segurança dos operadores (Salvador et al., 2018; Marchiori \& Santos, 2015).

\subsection{Radiografia de tórax}

Com a disponibilidade de inúmeros exames complementares, a prática médica tem evoluído para diagnósticos cada vez mais rápidos e com margem de erro menor. Dessa forma, o estudo por imagem é essencial para a avaliação clínica e cirúrgica de diversas patologias (Prando \& Moreira, 2014).

Por conseguinte, o conhecimento sobre os parâmetros de normalidade da radiografia de tórax é essencial para o diagnóstico de possíveis alterações. Através desse exame é possível identificar as principais estruturas do tórax que podem ser divididas em parede torácica (ossos e partes moles), coração, cavidades pleurais, vias aéreas, hilos pulmonares, vasculatura pulmonar, mediastino, aorta, pulmões direito e esquerdo (Wada et al., 2019).

Além disso, a análise da técnica utilizada na imagem que está sendo analisada é fundamental. A imagem obtida pelo raio x de tórax padronizada é realizada com o paciente em posição ortostática nas incidências póstero-anterior e perfil em inspiração profunda (Brant \& Helms, 2015). 
Nesse sentido, a incidência póstero-anterior torna-se mais fidedigna em relação à anteroposterior por conta da divergência dos feixes de raio x pois aproxima o coração do detector dos raios e reduz sua ampliação, além de retirar as escápulas do campo pulmonar (Hodler et al., 2019).

A partir disso, a compreensão da semiologia radiológica passa a ser essencial para a correta interpretação da radiografia torácica que, aliada à terminologia, descrição dos achados e clínica do paciente direcionam o profissional a um diagnóstico com mais segurança (Hodler et al., 2019).

Assim, há o desenvolvimento do olhar clínico para a identificação dos principais sinais radiológicos do tórax, os quais trazem maior especificidade às hipóteses levantadas na investigação e que culminam no estreitamento do diagnóstico diferencial (Hodler et al., 2019).

\subsection{Principais utilizações}

A radiografia de tórax é amplamente utilizada na prática médica. Equivale ao exame de imagem mais solicitado para pacientes com dor torácica. Isso se deve a algumas características do mesmo, tais como, a praticidade na execução do exame, o baixo custo em relação aos demais e a velocidade na obtenção da imagem (Wada et al., 2019b).

Além disso, este exame é utilizado rotineiramente em pacientes que necessitam de cuidados intensivos, isto é, pacientes que precisam de suporte ventilatório, ou os que utilizam sondas para alimentação, e/ ou aqueles que possuem acesso venoso central. Órgãos regulamentadores da prática médica preconizam a utilização diária desse exame em unidades de terapia intensiva, a fim de certificar o posicionamento correto destes aparatos médicos, evitando assim, possíveis complicações causadas pelos mesmos (Wada et al., 2019c).

Em continuidade a utilização do raio $\mathrm{X}$ de tórax, tem-se a pneumonia, uma doença que é causada pelo acúmulo de secreção no parênquima pulmonar, seguido de um processo inflamatório que compromete em diferentes graus a função respiratória, podendo gerar quadros de síndrome do desconforto respiratório agudo, edema pulmonar bilateral e hipoxemia arterial (Mizgerd et al., 2018).

Acerca dos sinais radiográficos encontrados na pneumonia, temos alguns sinais que sugerem tal enfermidade, tais como opacificação predominante basal dos pulmões e perda de volume pulmonar. Entretanto para a confirmação diagnóstica bem como uma melhor avaliação do estado geral do doente, a tomografia de tórax é o exame de escolha (Hodler et al., 2019).

Outra enfermidade em que radiografia de tórax se faz muito presente consiste na insuficiência cardíaca congestiva. Essa síndrome consiste na incapacidade do coração de bombear sangue para o resto do corpo, resultando em uma congestão pulmonar, podendo até se tornar uma congestão sistémica. Os principais sintomas encontrados em pacientes com tal morbidade são dispneia, ortopneia, dispneia paroxística noturna, isto é, falta de ar associada a sensação de afogamento que acontece durante o sono (Departamento de Insuficiência Cardíaca [DEIC], Sociedade Brasileira de Cardiologia [SBC], 2018).

Em relação aos achados radiográficos é possível citar a cardiomegalia e sinais de edema pulmonar, isto é, a trama vascular é mais perceptível. Entretanto é importante salientar que um raio X de tórax sem alterações não descarta a possibilidade do paciente possuir a doença (DEIC \& SBC, 2018).

Ainda relacionado as últimas enfermidades é possível afirmar que as duas podem compartilhar um achado em comum na radiografia de tórax, trata-se de um derrame pleural. Esse sinal corresponde ao acúmulo de líquido entre as duas membranas pleurais, a pleura mais externa, chamada de parietal e a pleura mais interna, conhecida como visceral (Dantas \& Reis, 2018).

Prosseguindo com as melhores aplicações da radiografia torácica, faz-se presente o pneumotórax, doença causada pela presença de gás no espaço pleural, podendo esta ocorrer espontaneamente, por doença pulmonar prévia ou subsequente a um trauma. O principal sintoma encontrado nessa enfermidade é dor na região torácica que é dependente da respiração, isto é, uma dor que tem piora com os movimentos respiratórios (Rodrigues, 2019). 
O pneumotórax resulta em uma radiografia com o sinal do sulco profundo, ou seja, o seio costofrênico lateral do pulmão acometido possui uma transparência maior, assim o avaliador visualiza uma trama vascular pulmonar diminuída (Wada et al., 2019c).

Por fim, no contexto vivido pelo mundo atual, deve-se mencionar o papel da radiografia de tórax na infecção por covid19. Mesmo possuindo baixa sensibilidade na avaliação clínica de pacientes infectados por SARS-CoV-2, ela possui um papel no combate à patologia. Este exame, por ter mais praticidade na sua realização, bem como um menor custo em relação aos demais exames de imagem, pode ser solicitado em alguns casos, sendo a principal justificativa avaliar o tubo traqueal após intubação orotraqueal (Meirelles, 2020).

Ademais existem outras utilizações deste método, que seriam pacientes sem confirmação dessa enfermidade, locais que não tem acesso a tomografia de tórax e avaliar possíveis complicações trazidas pelo COVID-19 (Meirelles, 2020).

\subsection{Importância na formação acadêmica}

A interpretação radiográfica é desafiadora para os estudantes do curso de medicina que estão tendo seu primeiro contato com as imagens radiológicas, sendo passível o erro interpretativo e o diagnóstico por imagem. A partir disso, comprovou-se que a sistematização da interpretação da imagem pelos estudantes, os quais seguem uma lista de verificação da radiografia torácica, é fundamental para o aprendizado radiológico destes futuros profissionais (Kok et al., 2017).

Segundo Cheung et al. (2018), em um estudo comparativo com um grupo de estudantes de medicina do último ano e médicos recém-formados na Austrália, foi identificado a baixa acurácia no diagnóstico por imagem de diversas patologias, dentre elas pneumotórax, derrame pleural, massa pulmonar, etc. Além destas, uma radiografia de tórax dentro dos padrões de normalidade foi inserida na avaliação, cerca de $67 \%$ dos estudantes conseguiram interpretar a imagem como tal.

Dessa forma, é fundamental para a curva de aprendizado dos estudantes o desenvolvimento de métodos que facilitem o estudo da imagem para que a margem de erro de interpretação seja a menor possível (Kok et al., 2017).

Logo, ainda que uma pequena parcela dos profissionais médicos se torne radiologista, o domínio do conhecimento radiológico para o diagnóstico das principais patologias é indispensável para a formação médica, ainda mais para aqueles que atuam em serviços de urgência e emergência (Mehdipoor et al., 2017).

\section{Metodologia}

\subsection{Métodos de pesquisa}

O projeto consiste em um estudo transversal, descritivo e de abordagem quantitativa. Isto é, os pesquisadores por meio da elaboração de um questionário via Google Forms®, buscaram por meio da quantificação, avaliar os conhecimentos técnicos e práticos dos internos de medicina acerca do raio $\mathrm{X}$ de tórax.

\subsection{Procedimentos éticos}

O estudo será desenvolvido de acordo com a Resolução 466/2012 do Conselho Nacional de Saúde, que além de definir diretrizes e normas também regulamenta pesquisas científicas. A pesquisa será iniciada somente após submissão e consequente aceita pela Plataforma Brasil associado a liberação do mesmo pelo CEP - UNINOVAFAPI.

\subsection{Cenário e coleta de dados}

A pesquisa será realizada em Teresina - PI por meio de questionário realizado na plataforma Google Forms ${ }^{\circledR}$, o qual irá gerar um link, que será enviado aos estudantes através de mídias sociais ou e-mail. O método foi escolhido devido a pandemia vivencia nos anos de 2019, 2020 e 2021 e visando evitar a exposição dos pesquisadores. 
Os dados serão coletados a partir de um questionário elaborado pelos próprios pesquisadores, cujo conteúdo versará sobre conceitos sobre física das radiações e aspectos técnicos na avaliação da imagem.

\subsection{Período de estudo}

A coleta de dados será realizada no período de 20 de agosto até 30 de setembro de 2021.

\subsection{Participantes da pesquisa}

\subsubsection{Critérios de inclusão}

Os critérios que serão adotados para inclusão no estudo são: alunos com vínculo ativo com a instituição UNINOVAFAPI da cidade de Teresina-PI, cursando do $9^{\circ}$ ao $12^{\circ}$ período de medicina durante o ano de 2021.

\subsubsection{Critérios de exclusão}

Serão excluídos acadêmicos que concluíram a graduação até o desfecho da coleta de dados.

\subsection{Amostra e análise de dados}

\subsubsection{Amostra}

A amostra para o estudo constará de 139 estudantes do $9^{\circ}$ ao $12^{\circ}$ período do Curso de Medicina da UNINOVAFAPI, selecionados aleatoriamente entre os 215 matriculados no $1^{\circ}$ semestre de 2021. Para encontrar esse número foi usada a fórmula aplicável a populações finitas:

$$
\mathrm{n}=\frac{Z^{2} \cdot P(1-P) \cdot N}{E^{2} \cdot(N-1)+Z^{2} \cdot P(1-P)}=\frac{1,96^{2} \cdot 0,5 \cdot 0,5 \cdot 215}{0,05^{2} \cdot 214+1,96^{2} \cdot 0,5 \cdot 0,5}=139 \text { alunos }
$$

$\mathrm{Na}$ qual, (z) é o valor crítico, (E) a margem de erro, (p) a proporção esperada e (N) o tamanho da população, considerando o grau de confiança de $95 \%(\mathrm{z}=1,96)$, margem de erro $\mathrm{E}=5 \%$, proporção $\mathrm{p}=0,5$ e $\mathrm{N}=215$.

\subsubsection{Análise de dados}

Os dados coletados serão submetidos a uma análise estatística descritiva através das frequências absolutas e relativas. O processamento será feito por meio da planilha Excel e do Programa SPSS $®$. Os resultados serão apresentados em forma de tabelas e gráficos.

\subsection{Riscos e benefícios}

\subsubsection{Riscos}

A realização da pesquisa oferece riscos de grau mínimo de origem psicológica, intelectual e/ou emocional - relativos à possibilidade de constrangimento ao responder o questionário; desconforto; estresse. Os riscos serão minimizados uma vez que o participante não será identificado e os pesquisadores manterão os dados relativos à pesquisa em ambiente virtual seguro, visto que o Google Forms $®$ não fornece IP (Endereço de Protocolo da Internet) dos participantes, garantindo segurança, não violação e a integridade dos documentos virtuais. Assim, apenas os pesquisadores utilizarão os dados para realização da pesquisa, garantindo confidencialidade e anonimato.

\subsubsection{Benefícios}

Possíveis melhorias nos conhecimentos acerca do reconhecimento dos padrões de normalidade da radiografia de tórax, bem como uma possível melhora na maneira em que a disciplina de radiologia é ministrada no curso de medicina da instituição 
UNINOVAFAPI, localizada em Teresina - PI.

\section{Resultados}

A pesquisa apresentou resultados respaldados pelo Comitê de Ética em Pesquisa do Centro Universitário UNINOVAFAPI $n^{\circ}$ 44862521.0.0000.5210. Os participantes contribuíram de forma voluntária, além de manter os seus dados pessoais em completo sigilo. O estudo contou com a participação de 157 estudantes, os quais estavam distribuídos do $9^{\circ}$ ao $12^{\circ}$ período do Curso de Medicina da UNINOVAFAPI. A totalidade dos participantes estava de acordo com os termos da pesquisa, compreendendo e concordando com o Termo de Consentimento Livre Esclarecido.

O questionário dispunha de 9 perguntas, dessa forma a pontuação apresentou uma variação de 1 a 9 pontos. Pois nenhum dos participantes zerou o questionário. A média aritmética calculada a partir dos resultados foi de 6 pontos, o que equivale a $66,6 \%$ da prova. E a mediana de 7 pontos.

Figura 1: Distribuição do total de pontos marcados por número de participantes.

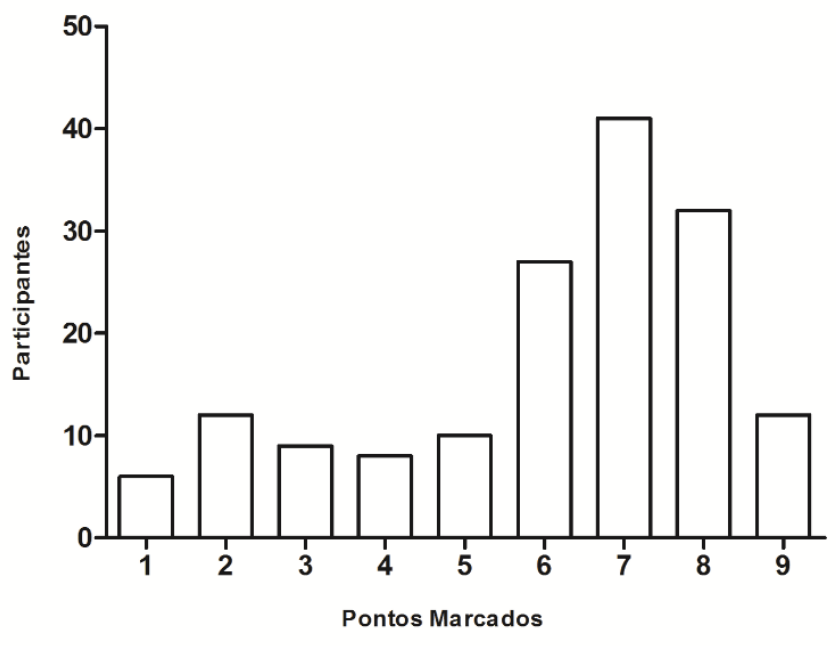

Fonte: Autores.

A Figura 1 apresenta a distribuição do total de pontos marcados por número de participantes, onde 6 participantes $(3,82 \%)$ obtiveram a pontuação de 1 ponto, 12 participantes $(7,64 \%)$ marcaram 2 pontos, 9 estudantes $(5,73 \%)$ acertaram 3 questões, 8 participantes $(5,1 \%)$ marcaram 4 pontos, 10 estudantes $(6,36 \%)$ obtiveram 5 pontos. Dessa forma, 45 estudantes (28,66\%) obtiveram uma pontuação abaixo da média.

Já 27 estudantes (17,2\%) obtiveram a pontuação de 6 pontos, igual a média, 41 participantes $(26,11 \%)$ marcaram 7 pontos, destacando-se como o grupo majoritário dentro da pesquisa, 32 participantes (20,38\%) acertaram 8 questões e 12 estudantes $(7,64 \%)$ obtiveram a pontuação máxima de 9 pontos. Totalizando 112 participantes $(71,33 \%)$ que tiveram uma pontuação igual ou acima da média (Figura 2). 
Figura 2: Porcentagem de participantes em relação à média aritmética.

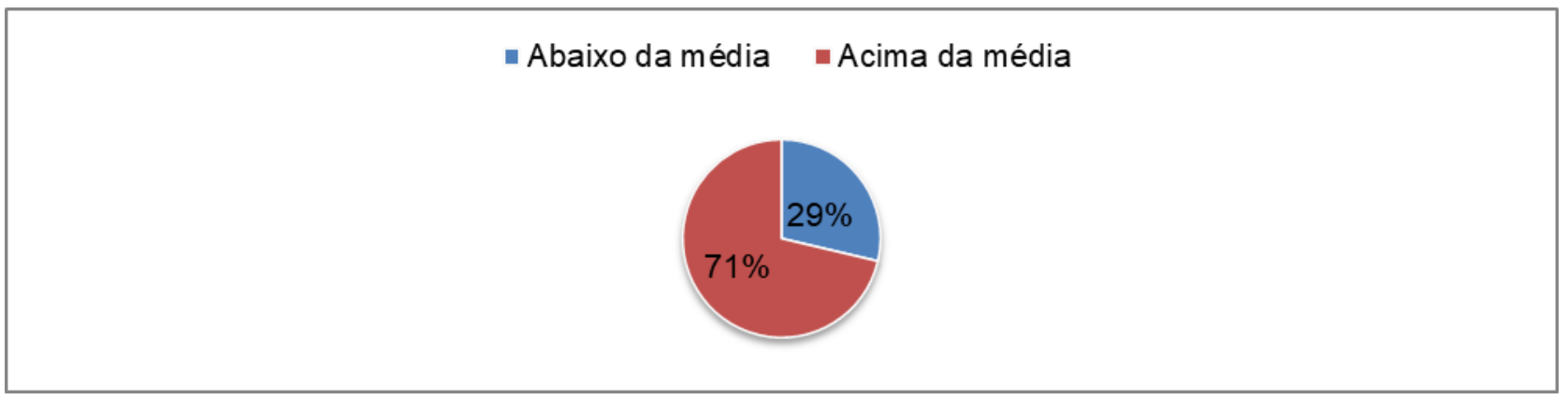

Fonte: Autores.

Todas as questões obtiveram um percentual de acerto maior que 50\%. A temática que alcançou maior êxito por parte dos participantes foi a respeito do princípio da radiografia. A questão "Como é produzida a radiografia?" teve 128 acertos, cerca de $81 \%$ dos estudantes. Tendo como resposta "os raios x emanam de uma pequena fonte e passam através de uma porção do corpo até um detector que grava os raios que o alcançam na forma de imagem”. As alternativas substituíam apenas o tipo de raio utilizado (Figura 3).

Figura 3: Distribuição das respostas em relação ao raio utilizado no princípio da radiografia.

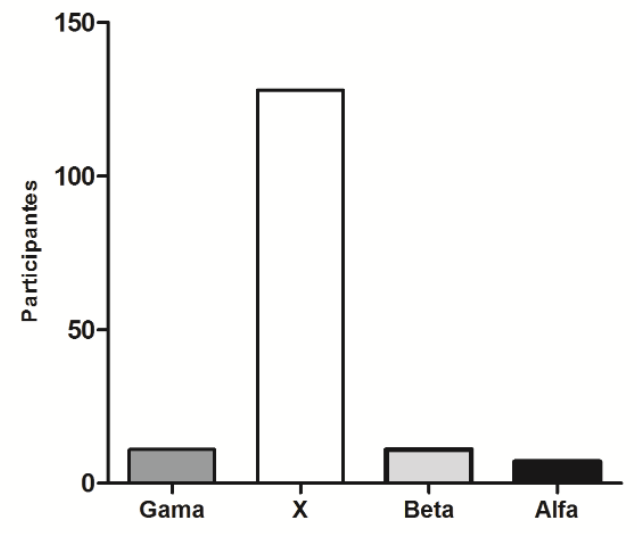

Fonte: Autores. 
Figura 4: Distribuição de respostas de como é verificado se o paciente fez a inspiração correta durante o exame.

- Exame realizado ao fim de uma inspiração profunda. De 9 a 10 costelas posteriores ou 5 a 6 anteriores e devem ser visualizadas.

- Exame realizado ao fim de uma expiração profunda. De 5 a 7 costelas posteriores ou 2 a 4 anteriores e devem ser visualizadas

n Exame realizado ao fim de uma expiração profunda. De 9 a 10 costelas posteriores ou 5 a 6 e devem ser visualizadas.

- Exame realizado ao fim de uma inspiração profunda. De 5 a 7 costelas posteriores ou 2 a 4 anteriores e devem ser visualizadas.

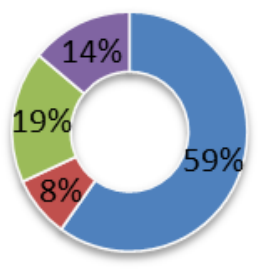

Fonte: Autores.

Por outro lado, as questões que os participantes tiveram o menor percentual de acertos, menos que $60 \%$ dos participantes pontuaram, estão relacionadas com a reprodução dos métodos e a rotina do diagnóstico radiológico. A questão “Como verificar se o paciente fez a inspiração correta durante o exame?" Tendo como resposta "Normalmente, os raios x frontais de tórax são realizados ao fim de uma inspiração profunda. De 9 a 10 costelas posteriores ou 5 a 6 anteriores e devem ser visualizadas à direita, acima da cúpula diafragmática direita, se o paciente inspirou adequadamente". As opções alternavam o número de costelas posteriores e anteriores e se o paciente estaria em processo de inspiração ou expiração. Apenas 94 (59\%) participantes responderam de forma correta (Figura 4).

A questão com menor índice de acertos foi “Qual a importância da radiografia de tórax em perfil?” onde apenas 79 dos participantes (51\%) marcaram como correto "Os objetos podem ser visualizados em três dimensões, aproximadamente 10\% das partes pulmonares são melhores ou exclusivamente visualizadas nos raios x em perfil e os exames em perfil podem confirmar a presença de lesão suspeitada na radiografia frontal.” A figura 5 apresenta o percentual das respostas, onde as opções alternam a respeito de quantas dimensões podem ser visualizadas na radiografia em perfil e se tal exame possui importância clínica.

Figura 5: Distribuição de respostas a respeito da importância da radiografia de tórax em perfil.

\footnotetext{
- Os objetos podem ser visualizados em três dimensões, exames em perfil podem confirmar a presença de lesão suspeitada na radiografia frontal.

- Os objetos podem ser visualizados em duas dimensões, os exames em perfil podem confirmar a presença de lesão suspeitada na radiografia frontal.

n Os objetos podem ser visualizados em três dimensões, os exames em perfil não tem importância diagnóstica
}

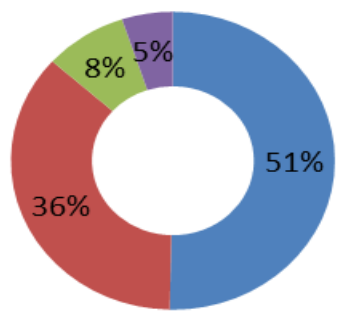

Fonte: Autores. 


\section{Discussão}

A radiografia simples do tórax possibilita a identificação de um conjunto de estruturas e órgãos, abrangendo os elementos da parede torácica, como ossos e partes moles, cavidades pleurais, pulmões, coração, aorta, vias aéreas, hilos pulmonares e mediastino. Portanto, é um exame que permite a identificação das principais estruturas anatômicas do tórax, entretanto, é necessário ter uma atenção especial ao emprego da técnica, as características do paciente e as variantes anatômicas (Wada et al., 2019).

Dentro deste contexto, a interpretação das imagens radiológicas é um processo cognitivo complexo, compreendendo diversos conhecimentos e habilidades. O processo de interpretação inclui a retirada de informações das imagens e sua combinação com os dados do paciente além do conhecimento prévio, o que permite compreender e realizar inferências sobre o significado dos achados (Barros, et al., 2019).

No presente estudo, os resultados apresentam índices relevantes na avaliação dos parâmetros de normalidade da radiografia de tórax. Considerando que média das pontuações obtidas pelos participantes foi de 6 pontos, o que equivale a $66,6 \%$ da prova. O resultado foi considerado satisfatório, pois 71,33\% dos participantes tiveram uma pontuação igual ou acima da média. Todas as questões obtiveram um percentual de acerto maior que $50 \%$.

Por outro lado, é importante dar destaque as questões com o menor percentual de acertos, onde menos que $60 \%$ dos participantes pontuaram. As questões que não obtiveram um resultado satisfatório estão relacionadas com a reprodução dos métodos e a rotina do diagnóstico radiológico. Tal resultado pode ser explicado pela a participação de alunos de diversos períodos, não só do último ano de curso. Pois a maioria dos estudos demonstram melhores resultados no conhecimento de radiografia de tórax em grupos com o maior nível de treinamento. (Eisen et al., 2006)

Em um estudo prévio realizado com estudantes de medicina da Universidade Federal do Rio de Janeiro, diferentemente da pesquisa realizada que avaliou os parâmetros de normalidade da radiografia de tórax, teve como objetivo avaliar a competência dos estudantes na interpretação de radiografias de tórax para o diagnóstico de tuberculose. Os resultados apontaram uma sensibilidade para o diagnóstico radiológico de $86,5 \%$ a $94,2 \%$, e a especificidade foi de $42 \%$ a $90 \%$, dependendo do nível de lesão analisada. Considerando que os estudantes tiveram um bom treinamento formal em radiologia (Silva et al., 2010).

O estudo de Samara e colaboradores (2021) trabalhou no mesmo sentido, objetivando avaliar o conhecimento e a acurácia de estudantes de medicina no diagnóstico de condições de emergência com risco de vida em radiografias de tórax, utilizando questionário eletrônico. Totalizando 530 participantes, todos os participantes responderam corretamente a pelo menos seis das sete questões, destes, $139(26,2 \%)$ participantes responderam todas as questões corretamente. Demonstrando um bom conhecimento com altos níveis de confiança no diagnóstico por parte dos estudantes.

Em estudo semelhante, que também utilizou de questionário de múltipla escolha, objetivava determinar se os estudantes de medicina atendiam aos padrões mínimos de diagnóstico utilizando radiografia de tórax. Seus resultados foram divergentes, 22 participantes completaram o estudo, dos quais apenas $51 \%$ responderam corretamente o diagnóstico. Dessa forma, não atenderam aos requisitos mínimos para habilidades de diagnóstico radiológico (Christiansen et al., 2014).

A interpretação correta das radiografias de tórax é essencial para tomada de decisões clinicas. Portanto, interpretações inadequadas têm implicações potenciais para o atendimento ao paciente. No entanto, o conhecimento sobre radiografia de tórax seja de grande importância em diversos âmbitos da prática médica, o treinamento formal varia amplamente. $\mathrm{O}$ ensino apresenta uma grande variabilidade entre os programas de treinamento em residência médica e faculdade de medicina (Geel et al., 2019; Jimah et al., 2020). 


\section{Conclusão}

No presente estudo, os resultados apresentam índices relevantes na avaliação dos parâmetros de normalidade da radiografia de tórax por estudantes de medicina. O resultado foi considerado satisfatório, pois 71,33\% dos participantes tiveram uma pontuação igual ou acima da média.

Todas as questões obtiveram um percentual de acerto maior que 50\%. Entretanto, as questões que não obtiveram um resultado satisfatório estão relacionadas com a reprodução dos métodos e a rotina do diagnóstico radiológico. Tal resultado pode ser explicado pela participação de alunos de diversos períodos, não só do último ano de curso. Havendo uma tendência de melhor resultado em grupos com o maior nível de treinamento.

Embora o conhecimento sobre radiografia de tórax seja de grande importância em diversos âmbitos da prática médica, o treinamento formal varia amplamente. Concluindo que ensino apresenta uma grande variabilidade entre os programas de treinamento em residência médica e faculdade de medicina.

\section{Referências}

Barros, P. et al. (2019). Worked Example X Aula Expositiva no aprendizado de Radiologia de estudantes de medicina. Dissertação de mestrado, Universidade Federal de Minas Gerais, Belo Horizonte, MG, Brasil. https://repositorio.ufmg.br/handle/1843/38739.

Brant, W. E. \& Helms, C. A. (2015). Fundamentos de Radiologia: Diagnóstico por Imagem; 4ed. Rio de Janeiro: Guanabara Koogan.

Cerri, G. G., Leite, C. C. \& Rocha, M. S. (2017). Tratado de radiologia. Ed. Manole.

Chen, M. Y. M., Pope, T. L. \& Ott, D. J. (2012) Radiologia básica; (2a ed.). AMGH.

Cheung, T. et al. (2018). Low accuracy and confidence in chest radiograph interpretation amongst junior doctors and medical students: Brief Communications. Internal Medicine Journal. 48(7), 864-8.

Christiansen, J. M., Gerke, O., Karstoft, J., \& Andersen, P. E. (2014). Poor interpretation of chest X-rays by junior doctors. Danish medical journal, 61(7), A4875.

Dantas, G. C. \& Reis, R. C. (2018). Protocolo de abordagem de derrame pleural. Revista de Medicina da UFC, $58(2), 67$.

Departamento de Insuficiência Cardíaca; Sociedade Brasileira de Cardiologia (2018). Diretriz brasileira de insuficiência cardíaca crônica e aguda. Arquivos Brasileiros de Cardiologia, 111 (3), 436-539. https://doi.org/10.5935/abc.20180190.

Eisen, L. A., Berger, J. S., Hegde, A., \& Schneider, R. F. (2006). Competency in chest radiography. A comparison of medical students, residents, and fellows. Journal of general internal medicine, 21(5), 460-465. https://doi.org/10.1111/j.1525-1497.2006.00427.x.

Geel, K. V., Kok, E. M., Aldekhayel, A. D., Robben, S., \& van Merriënboer, J. (2019). Chest X-ray evaluation training: impact of normal and abnormal image ratio and instructional sequence. Medical education, 53(2), 153-164. https://doi.org/10.1111/medu.13756

Hodler, J., Kubik-Huch, R. A., \& von Schulthess, G. K. (Eds.). (2019). Diseases of the Chest, Breast, Heart and Vessels 2019-2022: Diagnostic and Interventional Imaging. Springer.

Jimah, B. B., Appiah, A. B., Sarkodie, B. D. \& Anim, D. (2020). Competency in Chest Radiography Interpretation by Junior Doctors and Final Year Medical Students at a Teaching Hospital. Radiology. Research and Practice, vol. 2020, Article ID 8861206, 7 páginas. https://doi.org/10.1155/2020/8861206.

Kok, E. M., Abed, A., \& Robben, S. (2017). Does the Use of a Checklist Help Medical Students in the Detection of Abnormalities on a Chest Radiograph?. Journal of digital imaging, 30(6), 726-731. https://doi.org/10.1007/s10278-017-9979-0.

Marchiori, E \& Santos, M. (2015). Introdução a radiologia; (2a ed.), Guanabara Koogan, 2015.

Mehdipoor, G., Salmani, F. \& Arjmand Shabestari, A. Survey of practitioners' competency for diagnosis of acute diseases manifest on chest X-ray. BMC Med Imaging 17, 49 (2017). https://doi.org/10.1186/s12880-017-0222-8.

Meirelles, G. S. P. (2020). COVID-19: a brief update for radiologists. Radiologia Brasileira 53(5). 320-328. https://doi.org/10.1590/0100-3984.2020.0074.

Nascimento, A. (2018). X, como raio X. Laboreal, 14 (1), 73-75. http://dx.doi.org/10.15667/laborealxiv0118an.

Prando, A. \& Moreira, F. A. (2014) Fundamentos de radiologia e diagnóstico por imagem. (2a ed.) Elsevier.

Quinton, L. J., Walkey, A. J., \& Mizgerd, J. P. (2018). Integrative Physiology of Pneumonia. Physiological reviews, 98(3), 1417-1464. https://doi.org/10.1152/physrev.00032.2017.

Rodrigues, F. S. J. (2019). Pneumotórax em doentes pediátricos: casuística e breve revisão. Mestrado em Medicina. Faculdade de Medicina, Universidade de Lisboa. Lisboa, Portugal. https://repositorio.ul.pt/handle/10451/42956. 
Research, Society and Development, v. 10, n. 17, e105101724509, 2021

(CC BY 4.0) | ISSN 2525-3409 | DOI: http://dx.doi.org/10.33448/rsd-v10i17.24509

Salvador H., de Oliveira P., Silva M. de O. A. da, \& Gomes M. das N. (2018). Ondas Eletromagnéticas no Espectro Raio x num Contexto da Ciência, Tecnologia e Sociedade. REVISTA CEREUS, 10(4), 172-181. http://ojs.unirg.edu.br/index.php/1/article/view/2380.

Samara, O., AlRyalat, S. A., Malkawi, L., Ali, M. B., Kilani, A., Alkhalaileh, Z., Alkhatib, L., Hamad, I., Alawneh, Y., Al-Ryalat, N., \& Ryalat, S. (2021). Assessment of final-year medical students' performance in diagnosing critical findings on chest X-ray. Emergency radiology, 28(2), 333-338. https://doi.org/10.1007/s10140-020-01893-z.

Silva, V. et al.(2010). Competência de estudantes de medicina seniores na interpretação de radiografias de tórax para o diagnóstico de tuberculose. Jornal Brasileiro de Pneumologia, v. 36(2), p. 190-196. https://www.jornaldepneumologia.com.br/details/728/en-US/competencia-de-estudantes-de-medicinaseniores-na-interpretacao-de-radiografias-de-torax-para-o-diagnostico-de-tuberculose.

Teixeira, C. V.; Massoni, N. T.; Vargas, G. S. (2017). Raios x: um tema instigante para a introdução da física moderna e contemporânea na sala de aula do ensino básico. Experiências em Ensino de Ciências, 12(2), 80-93. https://lume.ufrgs.br/handle/10183/159728.

Wada, D. T., Rodrigues, J. A. H. \& Santos, M. K. (2019) Anatomia normal da radiografia de tórax. Medicina (Ribeirão Preto Online), 52 (supl. 1), 17-29. https://www.revistas.usp.br/rmrp/article/view/154766.

Wada, D. T., Rodrigues, J. A. H. \& Santos, M. K. (2019b) Aspectos técnicos e roteiro de análise da radiografia de tórax. Medicina (Ribeirão Preto Online), 52 (supl. 1), 5-15. https://www.revistas.usp.br/rmrp/article/view/154763.

Wada, D. T., Rodrigues, J. A. H. \& Santos, M. K. (2019c) Sinais radiológicos no tórax. Medicina (Ribeirão Preto Online), 52(1), 45-56. https://www.revistas.usp.br/rmrp/article/view/154870.

Wada, D. T., Rodrigues, J. A. H. \& Santos, M. K. (2019d) Sondas, cateteres e outros aparatos médicos na radiografia de tórax. Medicina (Ribeirão Preto Online), 52(1), 57-71. https://www.revistas.usp.br/rmrp/article/view/154797. 